\title{
Gestational Diabetes: Comparison of Random and Fasting Plasma Glucose as Modalities of Screening
}

\author{
Authors: \\ ${ }^{*}$ Chibuike F. Chukwunyere, ${ }^{1}$ David O. Awonuga, ${ }^{1}$ Olubiyi F. Adesina, ${ }^{2}$ \\ Ifeoma C. Udenze 3 \\ 1. Department of Obstetrics and Gynecology, Federal Medical Centre Abeokuta, \\ Abeokuta, Nigeria \\ 2. Department of Medicine, Federal Medical Centre Abeokuta, Abeokuta, Nigeria \\ 3. Department of Clinical Pathology, College of Medicine, University of Lagos, \\ Lagos, Nigeria \\ *Correspondence to chibichuks@gmail.com
}

Disclosure: $\quad$ The authors have declared no conflicts of interest.

Received:

Accepted:

Keywords:

Citation:
22.05 .20

24.08.20

Fasting plasma glucose (FPG), gestational diabetes, oral glucose tolerance test (OGTT), random plasma glucose (RPG), screening.

EMJ Diabet. 2020;8[1]:110-117.

\section{Abstract}

Objective: Gestational diabetes is glucose intolerance of varying severity with onset in the index pregnancy. This study aimed to compare fasting plasma glucose (FPG) with random plasma glucose (RPG) among pregnant females as methods of screening for gestational diabetes.

Methods: A cross-sectional study of 100 pregnant females selected to have screening for gestational diabetes between gestational ages of 24 and 28 weeks using RPG and FPG. All the subjects had $75 \mathrm{~g}$ oral glucose tolerance test as the gold standard. Venous plasma glucose assay was performed using glucose oxidase method.

Results: The prevalence of gestational diabetes was $29 \%$ using FPG cut-off $\geq 5.1 \mathrm{mmol} / \mathrm{L}$ and $6 \%$ using RPG cut-off $\geq 7.8 \mathrm{mmol} / \mathrm{L}$. The RPG cut-off $\geq 11.1 \mathrm{mmol} / \mathrm{L}$ gave the lowest prevalence rate of $2 \%$, while $75 \mathrm{~g}$ oral glucose tolerance test (gold standard test) gave the highest prevalence rate of $30 \%$. RPG cut-off $\geq 7.8 \mathrm{mmol} / \mathrm{L}$ revealed a positive-predictive value of $66.7 \%$, negative-predictive value of $72.3 \%$, and area under the curve of 0.845 compared with FPG level at threshold of $5.1 \mathrm{mmol} / \mathrm{L}$, which gave positive-predictive value of $93.1 \%$, negative-predictive value of $95.8 \%$, and area under the curve 0.920 .

Conclusion: This study revealed that FPG threshold of $5.1 \mathrm{mmol} / \mathrm{L}$ alone performed excellently as a screening test.

\section{INTRODUCTION}

Gestational diabetes is defined as glucose intolerance of variable severity with onset or diagnosis made in the index pregnancy. ${ }^{1}$ Before

the Canadian physician Fredrick Banting and his medical student Charles Best discovered insulin in 1921, maternal and perinatal morbidities and mortalities associated with diabetes were vast. Despite advances in the diagnosis and treatment 
of gestational diabetes, there are still increased adverse perinatal outcomes.'

All pregnant females that have identifiable risk factors for gestational diabetes should be screened using fasting plasma glucose (FPG), random plasma glucose (RPG), and/or oral glucose tolerance test (OGTT). ${ }^{1}$ Recent studies reveal that FPG will be beneficial for gestational diabetes screening and may reduce the morbidities and mortalities associated with gestational diabetes. ${ }^{1-5}$

Recent studies suggest a trend towards rising cases of gestational diabetes, with a prevalence rate of $6-18 \%$ in an African population. ${ }^{7-12}$ In Nigeria, studies on gestational diabetes found prevalence rates from $4.9 \%$ to $13.9 \%$ at different antenatal populations. ${ }^{8,10,11}$ A study by Jesmin et al. ${ }^{10}$ found prevalence of gestational diabetes to be $9.7 \%$ according to World Health Organization (WHO) criteria, but was $12.9 \%$ according to American Diabetes Association (ADA) criteria. There is a need for studies on RPG and FPG in the authors' local settings that may give evidence towards formulation of protocols that can translate to better patient management. Available evidence suggests that screening for gestational diabetes within the pregnant population increases the detection of females affected by diabetes in pregnancy and thus improves maternal and perinatal outcomes. ${ }^{12-16 .}$

Presently, most laboratory tests for gestational diabetes do not meet the characteristics of screening tests set out by the UK National screening committee (UK NSC), a modified form of WHO criteria for screening tests, and therefore may not be completely adapted to resource-limited settings. ${ }^{6}$ This emphasises that a screening test should be simple, safe, precise, and have facilities for diagnosis and treatment. ${ }^{6}$ At present, laboratory screening for gestational diabetes is not part of a compulsory universal screening care in antenatal settings in the majority of low-resource settings. Although the current guidelines and recommendations used are adapted from high-incomes settings and may not be very cost effective and generally acceptable in the authors' local settings. Therefore, there is a need for an appropriate screening test that will be universally acceptable and applicable to all pregnant females in lowresource settings. There is possibly a link to cost in diagnosis, which presently limits the universal application of OGTT in low-resource settings and the larger population of females at risk within the population.

A few studies have investigated the significance of the new WHO criteria for diagnosis of gestational diabetes in low-resource settings between 24 and 28 weeks gestational age using FPG $\geq 5.1 \mathrm{mmol} / \mathrm{L}(92 \mathrm{mg} / \mathrm{dL})$ and/or 1-hour post OGTT $\geq 10.0 \mathrm{mmol} / \mathrm{L}(180 \mathrm{mg} / \mathrm{dL})$, and 2 hour $\geq 8.5 \mathrm{mmol} / \mathrm{L}$ (153 mg/dL) following $75 \mathrm{~g}$ OGTT glucose load with one or more abnormal value.

It is crucial to determine the prevalence of gestational diabetes, especially in a low-resource setting such as the authors', as well as to compare the accuracy of low-cost methods such as RPG and FPG in screening for gestational diabetes. Following the change from WHO 1999 to WHO 2013 criteria (the former was based on the maternal impaired glucose tolerance and the risk of the mother developing diabetes in the future, while the latter was based on the odds ratio of 1.75 for adverse neonatal outcomes), no local study in a low-resource setting has evaluated the usefulness of the new FPG cut-off value of $5.1 \mathrm{mmol} / \mathrm{L}$ (92 $\mathrm{mg} / \mathrm{dL})$ in screening of pregnant females for gestational diabetes, including its sensitivity and specificity as a screening tool. Due to the cost implication associated with OGTT, especially in resource-constrained settings, less invasive and less expensive screening tests such as FPG and RPG could be promising as screening test in this setting. Most females in resourceconstrained settings still do not receive routine OGTT in centres that practise universal screening for gestational diabetes.

There is paucity of data on the use of FPG for gestational diabetes screening. Despite the projected increase in prevalence of diabetes and gestational diabetes due to demographic transitions to westernised lifestyle, few investigations comparing FPG with existing methods of gestational diabetes screening have been completed. This study differs from the current literature by comparing FPG and RPG with the standard OGTT as modalities of screening for gestational diabetes and is novel with the aim to develop simpler screening tests for this. The authors adapted a cost-effective and applicable method of gestational diabetes screening in a low-resource setting that will 
help in the reduction of adverse maternal and perinatal outcomes associated with gestational diabetes. The objective of this study was to compare RPG and FPG as screening methods for gestational diabetes.

\section{MATERIALS AND METHODS}

This was a comparative, cross-sectional study among consecutive pregnant females attending antenatal care with at least one identifiable risk factor for gestational diabetes (as noted in the inclusion criteria).,11 The study subjects were recruited using a multistage probability sampling method to assess the screening of gestational diabetes comparing FPG and RPG. The sampling frame was all pregnant females who booked for antenatal care from April 2018 to December 2019 at Federal Medical Centre Abeokuta (FMCA), Abeokuta, Nigeria, between 24- and 28-weeks gestational age. FMCA offers specialised obstetric services to the population of pregnant females residing in Abeokuta community and its surrounding area. Abeokuta is mainly a civil service population comprising federal, state, and local government civil servants, teachers, different cadres of traders, and farmers. The religion composition comprises mainly Christians and Muslims, with a handful of other native traditional African religion practitioners.

The inclusion criteria identifiable risk factors for gestational diabetes were previous fetal macrosomia, birth weight $\geq 4.0 \mathrm{~kg}$, history of diabetes in first-degree relatives, BMI $\geq 30$ or booking $\mathrm{BMI} \geq 25$, history of unexplained perinatal loss or malformed infant, repeated mild glycosuria 1+ or an isolated heavy glycosuria $\geq 2+$, maternal age $\geq 35$ years, history of gestational diabetes or impaired glucose tolerance in previous pregnancies, chronic hypertension, pre-eclampsia and gestational hypertension, polyhydramnios, and larger-than-date uterus in singleton pregnancy.111 Exclusion criteria were pregnant females who did not consent to the study and those with no identifiable risk factor for gestational diabetes. Additionally, those with previous history of diabetes were excluded from the study.

The sample size of 100 participants were calculated using the formula to estimate the mean difference of a continuous outcome based on matched data, according to the formula described by Sullivan. ${ }^{7} \quad Z_{\alpha}=1.96$ for 95\% confidence interval, according to studies by Djelmis et al., ${ }^{18} 23.1 \%$ (1,074) of females were diagnosed with gestational diabetes according to implementation of the International Association of Diabetes and Pregnancy Study Groups (IADPSG) criteria. The multistage probability sampling was used to select 100 consenting individuals who met the inclusion criteria from the sampling frame of pregnant women attending antenatal care. The selected subjects had RPG, FPG, and OGTT. The sampling frame included all pregnant females attending antenatal care at FMCA. At the antenatal clinic of FMCA, there are three different units (Firm A, Firm B, Firm C) running their antenatal care at different days of the week: Firm A on Thursdays, Firm B on Mondays, and Firm C on Tuesdays. The sample size of 100 was shared using multistage sampling to recruit 38 participants from Firm A, 31 participants from Firm B, and 31 from Firm C. This was based on the ratio of the last 6 months of antenatal care attendance (July 2017 to December 2017) of 1,156 pregnant subjects in Firm A, 1,428 in Firm B, and 1,162 in Firm C, giving a ratio of $31 \%, 38 \%$, and $31 \%$, respectively.

The bio-data and brief information, such as gestational age, parity, previous history of gestational diabetes, and diabetes, were obtained from the subjects who consented to the study. They had RPG at contact (having ensured that the patient was not in a fasting state) $)^{19,20}$ and thereafter FPG and $75 \mathrm{~g}$ OGTT were scheduled to be performed during subsequent antenatal care visits between gestational age of 24 and 28 weeks. $^{19,20}$ Samples of venous blood were collected into sodium fluoride containers. There was no delay in separating the plasma and, usually, the sample analyses were performed expediently to prevent the breakdown of the glucose. The glucose oxidase method of estimation of plasma glucose was performed, which involved the use of glucose oxidase reacting with glucose, water, and oxygen to form gluconic acid and hydrogen peroxide. The hydrogen peroxide produced oxidises a chromogen or the consumption of oxygen measured to estimate the amount of glucose present. ${ }^{21}$

The diagnosis of gestational diabetes was made using at least one abnormal result using the WHO 2013 criteria. ${ }^{22}$ This included fasting $\geq 5.1 \mathrm{mmol} / \mathrm{L}$, 
1 hour $\geq 10.0 \mathrm{mmol} / \mathrm{L}, 2$ hour $\geq 8.3 \mathrm{mmol} / \mathrm{L}$, and 3 hour $\geq 7.8 \mathrm{mmol} / \mathrm{L}$. Positive screening test is considered as RPG $\geq 7.8 \mathrm{mmol} / \mathrm{L}$ and/or FPG $\geq 5.1$ $\mathrm{mmol} / \mathrm{L}$. The test of accuracy was calculated using sensitivity and specificity of the screening test compared to the gold standard.

The primary outcome was measured as the accuracy of RPG and FPG in screening of patients for gestational diabetes. Secondary outcome was the prevalence of gestational diabetes according to RPG, FPG, and OGTT. Limitation was that the study participants had at least one risk factor for gestational diabetes.

Data entry and analyses were performed using International Business Machines Statistical Package for Social Sciences (IBM SPSS) version 22. The data were presented as frequency tables and graphs with the continuous variables that are normally distributed presented as mean ( \pm standard deviation). Associations were tested using chi squared test for categorical variables and the differences in mean values using student $t$ test and analysis of variance (ANOVA) for continuous variables. Significance level were set at $p$ value $<0.05$. The accuracy of RPG and FPG were calculated using sensitivity and specificity as stated below. Receiver operating characteristics (ROC) curve was used to plot the probability of detecting gestational diabetes cases. Data analysis was conducted by the investigator with assistance of the medical statistician. Ethical clearance approval was given by the Health Research Ethics Committee of Federal Medical Centre, Abeokuta, Nigeria.

\section{RESULTS}

During this study, a total of 100 eligible pregnant females were screened for gestational diabetes using FPG, RPG, and the $75 \mathrm{~g}$ OGTT. The mean age \pm standard deviation of the participants was 34.81 \pm 4.04 years, mean BMI was $31.46 \pm 7.29$, and modal parity was $1(32 \%)$. The majority of the pregnant subjects (74\%) had tertiary level of education. The mean RPG of the participants was $5.53 \pm 1.57 \mathrm{mmol} / \mathrm{L}$, while the mean FPG was $4.70 \pm 1.02 \mathrm{mmol} / \mathrm{L}$. The prevalence of gestational diabetes was $29 \%$ using FPG cut-off $\geq 5.1 \mathrm{mmol} / \mathrm{L}$, $16 \%$ using FPG cut-off $\geq 5.3 \mathrm{mmol} / \mathrm{L}$, and $6 \%$ using RPG cut-off $\geq 7.8 \mathrm{mmol} / \mathrm{L}$. The RPG cut-off $\geq 11.1$ $\mathrm{mmol} / \mathrm{L}$ gave the lowest prevalence rate of $2 \%$, while $75 \mathrm{~g}$ OGTT gave the highest prevalence rate of $30 \%$. The percentage of females with positive test, sensitivity, specificity, and positiveand negative-predictive values for various FPG and RPG cut-off values are presented below. The FPG cut-off values between $5.1 \mathrm{mmol} / \mathrm{L}$ and $5.5 \mathrm{mmol} / \mathrm{L}$ classified $29 \%$ and $16 \%$ of the subjects, respectively, as having a positive test. The sensitivities decreased as cut-off values for FPG were increased, from $90.0 \%$ at $5.1 \mathrm{mmol} / \mathrm{L}$, to $43.3 \%$ at $5.5 \mathrm{mmol} / \mathrm{L}$, and $6.7 \%$ at $7.0 \mathrm{mmol} / \mathrm{L}$. Additionally, increasing the FPG cut-off from 5.1 $\mathrm{mmol} / \mathrm{L}$ to $5.5 \mathrm{mmol} / \mathrm{L}$ decreased the specificity from $97.1 \%$ to $95.7 \%$, while the efficiency of the test decreased from $95 \%$ to $80 \%$, respectively. The highest efficiency of the screening tests was 95\%; this was obtained at FPG cut-off value of $5.1 \mathrm{mmol} / \mathrm{L}$. The area under curve (AUC) was plotted for RPG with the gold standard OGTT test (Figure 1) giving AUC of 0.845, which can be classified as a good test with a statistically significant curve $(p=0.000)$. The ROC constructed in order to compare the ability of FPG with OGTT in differentiating between subjects with diagnosis of gestational diabetes gave AUC of 0.920, which can be classified as excellent (Figure 2).

\section{DISCUSSION}

The prevalence rate of gestational diabetes was $29 \%$ by using FPG cut-off value $5.1 \mathrm{mmol} / \mathrm{L}$, while $30 \%$ prevalence rate was obtained by using the standard $75 \mathrm{~g}$ OGTT. The findings from this study was higher than that by Mortensen et al. $^{23}$ in a prospective community study in Copenhagen, Denmark, in which they used a riskbased approach and clinical criteria for potential diabetes for screening. This study also found higher prevalence rate of gestational diabetes than that quoted by Djelmis et al. $^{18}$ in a cohort study of 4,646 pregnant females who underwent $75 \mathrm{~g}$ OGTT in Croatia. Djelmis et al. ${ }^{18}$ found the prevalence of gestational diabetes, according to IADPSG and National Institute for Health and Care Excellence (NICE) criteria, to be $23.1 \%(1,074)$ of gestational diabetes cases and $17.8 \%$ (826) of gestational diabetes cases, respectively. ${ }^{18}$ FPG levels of $5.1-5.5 \mathrm{mmol} / \mathrm{L}$ comprised 409 (8.8\%) of cases, while 50 (1.1\%) had overt diabetes. 


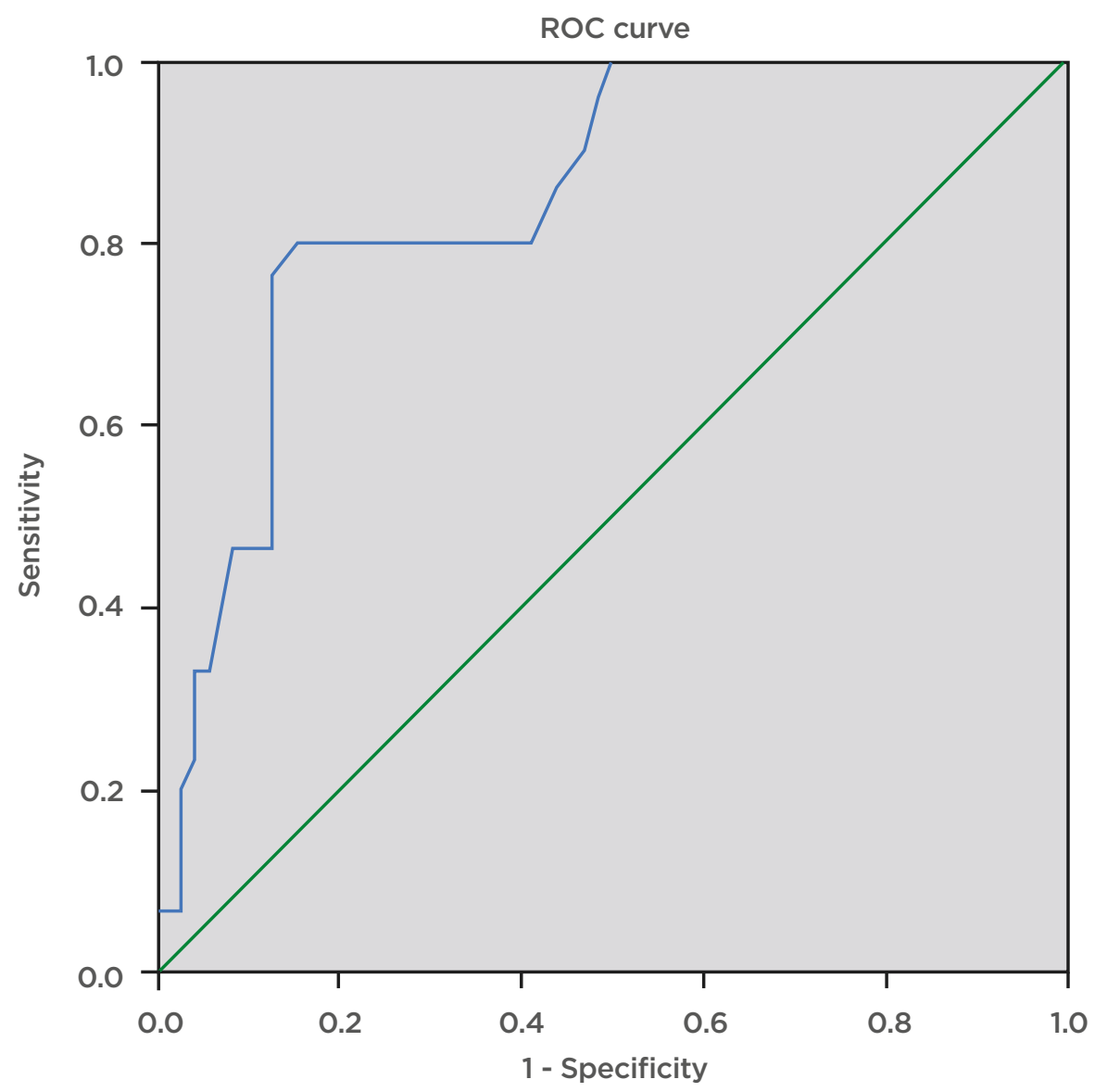

Diagonal segments are produced by ties.

Figure 1: Receiver operating characteristics curve for the accuracy of random plasma glucose $(\geq 7.8 \mathrm{mmol} / \mathrm{L})$ in prediction of gestational diabetes.

The ROC curve was constructed in order to compare the ability of random plasma glucose with the oral glucose tolerance test to differentiate between subjects with diagnosis of gestational diabetes. The area under curve was plotted for random plasma glucose with the gold standard oral glucose tolerance test which gave area under the curve of 0.845 , which can be classified as a good test with a statistically significant curve $(p=0.000)$.

ROC: receiver operating characteristics.

This is comparable to the findings in this study in which two (2.0\%) of the subjects had overt diabetes. The mean FPG found in this study was $4.70 \pm 1.02 \mathrm{mmol} / \mathrm{L}$, higher than $3.81 \pm 0.85 \mathrm{mmol} / \mathrm{L}$, quoted by Afolabi et al. ${ }^{24}$ in Lagos, Nigeria.

The accuracy of the screening tests for gestational diabetes in this study revealed FPG to have the high sensitivity of $90.0 \%$ and specificity of $97.1 \%$ at the cut-off value of $5.1 \mathrm{mmol} / \mathrm{L}$ and sensitivity of $60.0 \%$ and specificity of $97.1 \%$ at the threshold value of $5.3 \mathrm{mmol} / \mathrm{L}$. This is similar to the findings by Trujillo et al., ${ }^{3}$ who calculated a sensitivity of $96.9 \%$, although the FPG cut-off value used was $4.4 \mathrm{mmol} / \mathrm{L}$. Additionally, they observed a sensitivity of $92.0 \%$ at cut-off $4.7 \mathrm{mmol} / \mathrm{L}$. The higher sensitivity value reported by Trujillo et al. $^{3}$ was due to the low cut-off value of FPG used for gestational diabetes screening in that study. However, Trujillo et al. ${ }^{3}$ found a sensitivity of $86.8 \%$ at FPG cut-off value of $5.1 \mathrm{mmol} / \mathrm{L}$ (92 $\mathrm{mg} / \mathrm{dL}$ ), which is lower than the sensitivity found at this study (90.0\%) at the same FPG cutoff value of $5.1 \mathrm{mmol} / \mathrm{L}$. Cuscheri et al. ${ }^{20}$ found that RPG (sensitivity: 69.2\%; specificity: $43.3 \%$; AUC: 0.598; standard error: 0.36; $p=0.005$; 95\% confidence interval: 0.527-0.668) was an inferior predictor test when compared to FPG at an indicative predictor gestational diabetes cut-off point for FPG and RPG of $4.5 \mathrm{mmol} / \mathrm{L}$. Khan et al., ${ }^{19}$ 


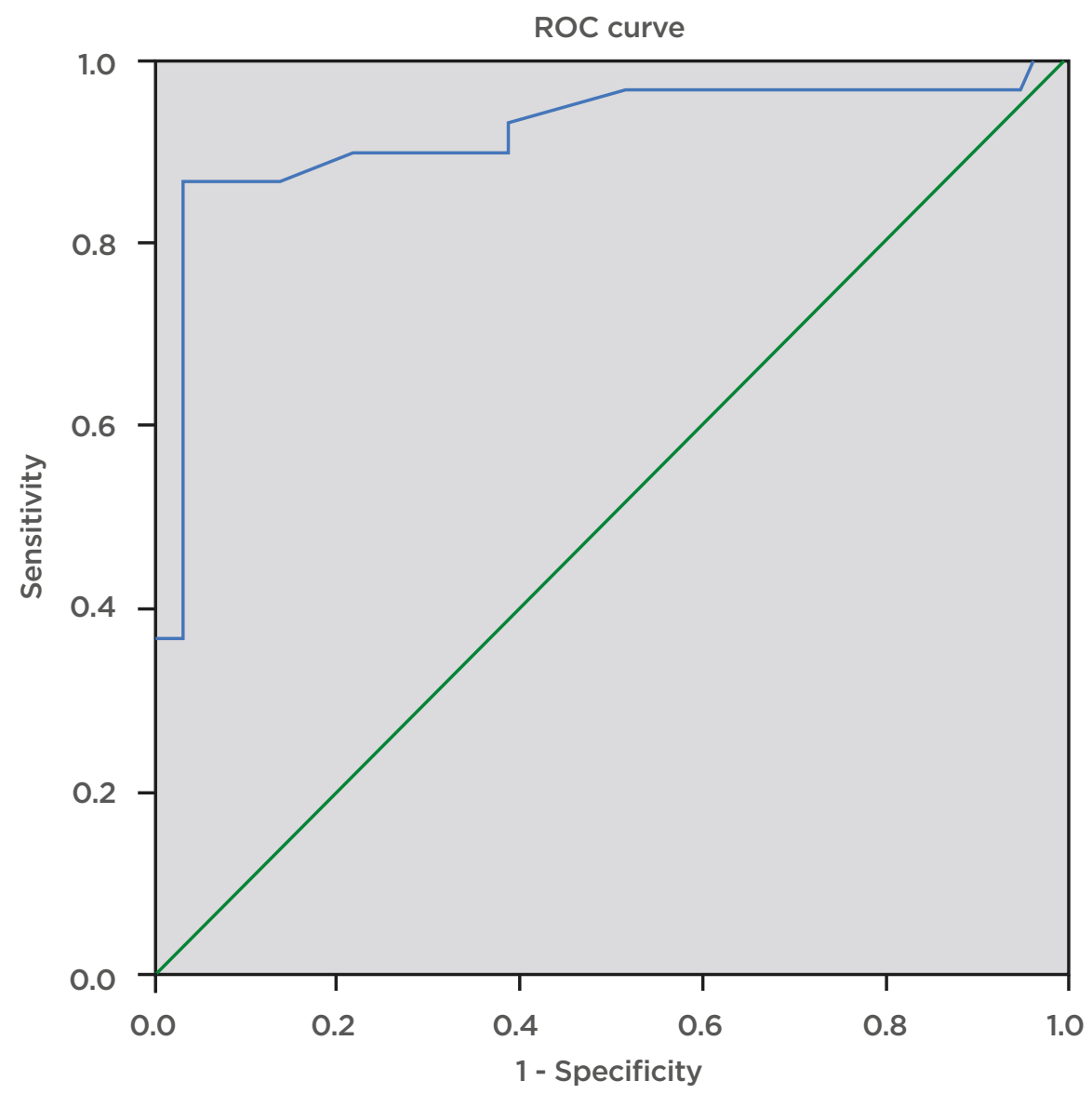

Diagonal segments are produced by ties.

Figure 2: Receiver operating characteristics curve for the accuracy of fasting plasma glucose (cut-off $\geq 5.1 \mathrm{mmol} / \mathrm{L}$ ) in prediction of gestational diabetes.

The ROC curve was constructed to compare the ability of fasting plasma glucose with the oral glucose tolerance test to differentiate between subjects with diagnosis of gestational diabetes. The ROC curve revealed area under the curve of 0.920, which can be classified as excellent.

ROC: receiver operating characteristics.

in a study in Karachi, Pakistan, found FPG cut-off of $5.1 \mathrm{mmol} / \mathrm{L}$ to be the most efficient investigation and gave sensitivity of $66.66 \%$ and specificity of $81.25 \%$. Agbozo et al. ${ }^{25}$ found a sensitivity of $68.0 \%$ for gestational diabetes screening using FPG threshold $\geq 5.1 \mathrm{mmol} / \mathrm{L}$, which is lower than the findings in this study. The highest specificity found in this study was at FPG threshold of $\geq 7.0 \mathrm{mmol} / \mathrm{L}$ and $\mathrm{RPG} \geq 11.0$ $\mathrm{mmol} / \mathrm{L}$, which gave specificity of $100.0 \%$. The FPG threshold $\geq 5.1 \mathrm{mmol} / \mathrm{L}$ gave a specificity of $97.1 \%$, which is lower than the specificity of $100.0 \%$ found in a study by Trujillo et al. ${ }^{3}$ at same FPG cut-off. Agbozo et al. ${ }^{25}$ found a specificity of $81.0 \%$ at FPG threshold of $\geq 5.1$ $\mathrm{mmol} / \mathrm{L}$, which is substantially lower than the specificity of $97.1 \%$ found by this study at the same threshold. Although both studies were performed in pregnant individuals, Agbozo et al. ${ }^{25}$ screened subjects using a universal approach when compared to the selective screening based on risk factors that was used in this study. This could have accounted for the higher specificity found in this study; however, the finding was higher than the positive-predictive value found in a study by Saeedi et al., ${ }^{26}$ which gave positive-predictive value of $78 \%$ at FPG threshold of $5.2 \mathrm{mmol} / \mathrm{L}$. However, Saeedi et al. ${ }^{26}$ screened a population of pregnant females with known risk factors for gestational diabetes and the study was carried out in Swedish population. Geographical variation in risk factors may be possible explanations for these differences observed. In a study by Mohan et al. ${ }^{27}$ in an 
Indian population, the authors found a positivepredictive value of $54.5 \%$ at RPG threshold of $7.8 \mathrm{mmol} / \mathrm{L}$, while Reyes-Muñoz et al.,28 in a study in a Mexican population using FPG threshold values of $4.5 \mathrm{mmol} / \mathrm{L}, 4.7 \mathrm{mmol} / \mathrm{L}$, and $5.0 \mathrm{mmol} / \mathrm{L}$, found positive-predictive values of $12 \%$ (9-15\%), $23 \%$ (18-28\%), and $64 \%$ (54-73\%), respectively.

The efficiency of the screening tests was found to be $95 \%$ at FPG threshold of $5.1 \mathrm{mmol} / \mathrm{L}, 86 \%$ at FPG threshold of $5.3 \mathrm{mmol} / \mathrm{L}$, and $72 \%$ at RPG threshold of $7.0 \mathrm{mmol} / \mathrm{L}$. This is higher than the result quoted by Bhavadharini et al. ${ }^{5}$ in Southern India, who found efficiency of $40 \%$ at RPG threshold of $7.7 \mathrm{mmol} / \mathrm{L}$.

The ROC curve plotted is comparable to AUC value of 0.960 for FPG found by Trujillo et al. ${ }^{3}$ in a Brazilian cohort study. The findings are also similar to the result of Rajab et al. ${ }^{4}$ in a Bahrain population, who found an AUC of 0.962 at FPG threshold of $5.6 \mathrm{mmol} / \mathrm{L}$. Agbozo et al. ${ }^{25}$ in a study at Volta region of Ghana found an AUC of $>0.8$ for FPG to be very good and AUC of 0.6 for RPG to be poor and therefore concluded that RPG was unnecessary for selective gestational diabetes screening. Saeedi et al. ${ }^{26}$ in a study in Swedish population found an AUC of 0.92 for FPG threshold of $5.0 \mathrm{mmol} / \mathrm{L}$ for gestational diabetes screening.

\section{CONCLUSION}

The findings from this study revealed that FPG threshold of $5.1 \mathrm{mmol} / \mathrm{L}$ has a high sensitivity of
90.0\% and specificity of $97.1 \%$, as well as AUC of 0.920 , which is excellent for a screening test. The use of RPG threshold of $7.8 \mathrm{mmol} / \mathrm{L}$ gave a sensitivity of $13.8 \%$ and specificity of $97.1 \%$ with an AUC of 0.845. FPG was superior to RPG in screening for gestational diabetes among pregnant females, and gave a prevalence rate of $29 \%$, close to the prevalence rate of $30 \%$ that was diagnosed with standard OGTT. The possibility of use of FPG alone for gestational diabetes screening as an alternative to OGTT can be considered in guidelines.

\section{RECOMMENDATION}

The findings from this study revealed that FPG alone is an excellent screening test for gestational diabetes and can be considered as an alternative to standard OGTT, especially in resourceconstrained settings where cost, facilities, and workforce, especially in the primary healthcare level, may hinder gestational diabetes screening using the standard OGTT.

\section{STATEMENT OF ETHICS}

Ethical approval was granted by the health ethics and research committee of the Federal Medical Centre Abeokuta with the protocol identification number FMCA/470/HREC/10/2016/07. NREC assigned number is NHREC/O8/10-2015 and federal wide assurance is US/REG NO: FWA/ Q0018660/02/28/2017. Written informed consent was signed and obtained from the subjects prior to their participation in the study.

\section{References}

1. Oputa RN, Nzeribe EA. Gestational diabetes mellitus: a clinical challenge in Africa. African J Diabetes Med. 2013:21:2;29-31.

2. Agarwal MM. Gestational diabetes mellitus: screening with fasting plasma glucose. World J Diabetes. 2016;7(14):279-89

3. Trujillo $\mathrm{J}$ et al. Fasting plasma glucose to avoid a full OGTT in the diagnosis of gestational diabetes. Diabetes Res Clin Pract. 2014;105(3):322-6.

4. Rajab EK et al. Screening for gestational diabetes by measuring fasting plasma glucose levels. J Sci Res Med Sci. 2003;5(1-2):5-8.

5. Bhavadharini B et al. Use of capillary blood glucose for screening for gestational diabetes mellitus in resource-constrained settings. Acta Diabetol. 2016;53:91-7.

6. UK National Screening Committee (UK NSC). Criteria for appraising the viability, effectiveness and appropriateness of a screening programme. 2015. Available at: https://www.gov.uk/government/ publications/evidence-review-criterianational-screening-programmes/ criteria-for-appraising-the-viabilityeffectiveness-and-appropriatenessof-a-screening-programme. Last accessed: 5 September 2020.

7. Mwanri AW et al. Prevalence of gestational diabetes mellitus in urban and rural Tanzania. Diabetes Res Clin Pract. 2014;103:71-8.

8. Anzaku AS, Musa J. Prevalence and associated risk factors for gestational diabetes in Jos, Northcentral, Nigeria. Arch Gynecol Obstet. 2013;287(5):859-63. 
9. Kuti MA et al. Oral glucose tolerance testing outcomes among women at high risk for gestational diabetes mellitus. J Clin Pathol. 2011;64(8):718-21.

10. Jesmin $\mathrm{S}$ et al. Screening for gestational diabetes mellitus and its prevalence in Bangladesh. Diabetes Res Clin Pract. 2014;103(1):57-62.

11. Fawole $\mathrm{AO}$ et al. Effectiveness of a structured checklist of risk factors in identifying pregnant women at risk of gestational diabetes mellitus: a crosssectional study. Niger J Clin Pract. 2014;17:495-501.

12. John $\mathrm{CO}$ et al. Foeto-maternal outcome of diabetes in a tertiary health facility in Nigeria. African J Diabetes Med. 2015;23(2):13-6.

13. Metzger BE, Coustan DR. Summary and recommendations of the Fourth International Workshop-Conference on Gestational Diabetes Mellitus. The Organizing Committee. Diabetes Care. 1998:21(Suppl 2):B161-7.

14. Jiwani A et al. Gestational diabetes mellitus: results from a survey of country prevalence and practices. J Maternal Fetal Neonatal Med. 2012;25:600-10.

15. Seshiah $\vee$ et al. Prevalence of gestational diabetes mellitus in South India (Tamil Nadu) - a community based study. J Assoc Physicians India. 2008:56:329-33.

16. Murphy A et al. "Diabetes Mellitus and Pregnancy", Decherney $\mathrm{AH}$ et al. (eds), Current Diagnosis and Treatment Obstetrics and Gynaecology (2013), 11 th $^{\text {th }}$ Edition, New York: McGrawHill, pp. 509-17.

17. Sullivan L. Power and sample size determination. 2020. Available at: https://sphweb.bumc.bu.edu/otlt/ MPH-Modules/BS/BS704_Power/. Last accessed: 5 September 2020.

18. Djelmis $\mathrm{J}$ et al. Prevalence of gestational diabetes mellitus according to IADPSG and NICE criteria. Int J Gynaecol Obstet. 2016;135(3):250-4.

19. Khan $\mathrm{SH}$ et al. Evaluation of fasting and random plasma glucose for diagnosis of gestational diabetes. J Coll Physicians Surg Pak. 2009;19(11):718-22.

20. Cuscheri $\mathrm{S}$ et al. The role of untimed blood glucose in screening for gestational diabetes mellitus in a high prevalent diabetic population. Scientifica. 2016;2016:1-6.

21. Dohnal $L$ et al. Comparison of three methods for determination of glucose. Prague Med Rep. 2010;111(1):42-54

22. World Health Organization (WHO). Diagnostic criteria and classification of hyperglycaemia first detected in pregnancy. 2013. Available at: https://apps.who.int/iris/ bitstream/handle/10665/85975/ WHO NMH_MND 13.2 eng.pdf;jsessionid $=9248 B 8 A 4841 \mathrm{CB} 72762 \mathrm{DEE}$ 69F444A4B67? sequence=1. Last accessed: 5 September 2020

23. Mortensen $\mathrm{HB}$ et al. A screening procedure for diabetes in pregnancy. Diabetes Metab. 1985;11:249-53.

24. Afolabi BB et al. Fasting plasma glucose levels in normal pregnant Nigerians. J Obstet Gynaecol. 2003;23(6):640-2

25. Agbozo F et al. Accuracy of glycosuria, random blood glucose, and risk factors as selective screening tools for gestational diabetes mellitus in comparison with universal diagnosing. BMJ Open Diabetes Res Care. 2018;6:e000493.

26. Saeedi M et al. Characteristics of different risk factors and fasting plasma glucose for identifying GDM when using IADPSG criteria: a crosssectional study. BMC Pregnancy Childbirth. 2018;18:225.

27. Mohan $\mathrm{V}$ et al. Comparison of screening for gestational diabetes mellitus by oral glucose tolerance tests done in the non-fasting (random) and fasting states. Acta Diabetol. 2014;51:1007-13

28. Reyes-Muñoz E et al. Sensitivity of fasting glucose for gestationa diabetes mellitus screening in Mexican adolescents based on International Association of Diabetes and Pregnancy Study Groups criteria: a diagnostic accuracy study based on retrospective data analysis. BMJ Open. 2018;8:e021617. 